\title{
USING CERAMIC CRAFTS TO TEACH USER CENTRED DESIGN FOR WATER PURIFICATION AND DEVELOPMENT
}

\author{
Richard HARLOW and Lyndon BUCK \\ Buckinghamshire New University, United Kingdom
}

\begin{abstract}
The development of more advanced technologies for manufacture and knowledge exchange has opened the world to wider trade and partnerships, requiring designers to consider the suitability of their products for cultures other than their own. With these advances a student's ability to access other cultures has also increased, allowing them to research a cultures practices and traditions in greater detail. However, the availability of certain technologies differs from country to country, with many developing countries having stable access to mobile telephones, while still lacking basic access to clean drinking water. The use of design for development briefs give students the opportunity to implement user centred design within a cultural context, and to select appropriate material processes for their intended areas of development. This paper explores the work produced by level five product design students completing the ceramic portion of their manufacturing module. The students were given a brief inspired by the authors' work in the EU Horizon 2020 funded WATERSPOUTT project. The students were asked to design and produce a ceramic filter and storage vessel for a developing area of their choice, taking into consideration the usability of the product, the cultural heritage of the area, and the local manufacturing resources available. The WATERSPOUTT project has worked to provide solar disinfection (SoDis) resources for the disinfection of local water for target communities in Uganda, South Africa, Ethiopia and Malawi. The authors' role in the project was to design a ceramic filter to be used in combination with solar disinfection technologies.
\end{abstract}

Keywords: Water filtration, ceramics design, user centred design, design for culture

\section{INTRODUCTION}

In higher education (HE) it is not uncommon for students to be set design projects with a wide scope in terms of the brief, materials and processes used. The primary concern of the design educator is fostering a student's ability to create a viable solution to a real-world problem, working with uncertainty and ambiguity, and to communicate the solution clearly through their final design outcome. While technology advances, the use of Computer Aided Design (CAD) and Computer Aided Manufacture (CAM) systems can ease the design process and increase the speed of concept development, it can however reduce a student's familiarity with materials and processes. Through manual manipulation of materials, a student can gain a deeper understanding of their properties, and more than this experience the user's tactile and emotional experience of the finished material [1]. Traditional ceramic processes improve a student's hand eye coordination and provide a tactile and observational sense of scale. These processes also act as a rapid tool for design visualization, allowing students to quickly experiment with basic forms and expand on their ideas with relative ease [2].

These experiences inform the designer of the visceral impacts of their products and allow them the opportunity to sample a range of material finishes for future concept development. In conventional design projects materials are selected at the discretion of the designer, though these choices may have an unconscious personal or cultural influence. While the perceptions of materials can differ among users, the selection of materials can be used to influence the user's feelings towards that product and create a greater emotional connection [1]. While tools exist to explore users' perceptions of material types, the timeframe allocated for the project described in this paper was not sufficient to explore these perceptions suitably. Instead the module encouraged students to focus on the application of materials and aesthetic styles that have been well established in the student's culture of choice. This approach was selected to 
encourage students to apply to Norman's three levels of processing, combining the local aesthetic to address the Viceral level, the usability and functionality required in the Behavioural level, and the material and cultural attachment applicable to the Reflective level [3]. While users overall may have similar reactions and physical responses to certain textures, materials and colours, each user can perceive these though a subjective or cultural perspective essentially changing their view of the product $[1,4]$. This paper discusses the work of level five students during the ceramic portion of their manufacturing module. Conventionally ceramics projects have centred around the production of household crockery or sculptural pieces, often with the intention of exploring the form of the object. This module aimed to incorporate the findings of Harlow and Buck [4] and Pedgley et.al. [5] by combining the material learning of the student, the user centred considerations in material, aesthetic and performance qualities, and finally the technical aspect of the water filters. Through this process student were given the opportunity to explore traditional craft methods such as coiling or press moulding along with contemporary technologies such as 3D printers. As a result of this students can judge the suitability of designs and production methods based on a range of traditional and technical knowledge sets developed during the module. The student brief was inspired by the authors work on the Horizon 2020 WATERSPOUTT project. WATERSPOUTT (Water - Sustainable Point-Of-Use Treatment Technologies) is an EU funded project through the Horizon 2020 programme which consists of 18 partners in 12 countries (Ireland, Malawi, Ethiopia, Uganda, South Africa, UK, Spain, Netherlands, Austria, Italy, Switzerland, Turkey). The main aim of the project is to design, develop and then pilot solar based technologies for treating drinking water at the point of use. These will be piloted and assessed for their impact on waterborne disease in Malawi, Ethiopia, Uganda and South Africa.

\section{CERAMIC DESIGN FOR DEVELOPING REGIONS}

When designing for developing regions those working to produce viable solutions will be met with a multitude of constraints including locally available materials and production skills, limited resources for further manufacture or the import of parts, limited waste management infrastructure, differing cultural beliefs and practices, different environments for product use, and stakeholders with alternative values to those of the designer [4]. While it would be unrealistic to expect students in an undergraduate course to tackle all these issues simultaneously, it is important to give students the opportunity to become aware of these potential barriers. By introducing a design brief for developing areas, students are given the opportunity to not only consider the factors raised above, but to take themselves out of the cultural and industrial design setting they are familiar with, and consider the needs of a user from an ethical standpoint rather that one of generating profit [6]. In these cases, students are challenged to focus on the health impacts of their design on users ranging in age, physical condition, and levels of societal development. Furthermore, incorporating usability, lifestyle demands, aesthetics, culture and local resources available to user that would have otherwise not been included in conventional design brief. These skills can enable students to grasp a deeper understanding of their own skillset and prepare them for a wider range of rolls post-graduation [4].

Ideally, the designer would be given the opportunity to visit the area their final design would be intended to be implemented. This would allow the designer first-hand knowledge of the environment and conditions the product would need to function within. More importantly this allows the designer the opportunity to interact with the user, identifying their needs, habits and aspirations [7]. Due to time and funding constraints this was not possible limiting students to desk based research for data collection. The students were given six weeks for the development and production of their filters and storage vessels. Due to the slow nature of ceramics production the students were often required to wait up to a week for models to dry suitably for firing, with an additional two days wait when firing.

The group of level five students were set the following brief:

"Design and produce a water filter housed in a ceramic vessel to collect the filtered water being produced. For the design to be accepted it should be catered to the culture of the intended user. The selection of the country you will be designing for will be selected by you. Emphasis should be placed on the usability of the filter and accessibility of the filtered water, while also communicating the forms, and styles and manufacturing processes of the intended culture for adoption". 
The constraints applied by the projects brief primarily apply to the function of the product, with the styling and form being left open to the interpretation of the student's research. The introduction of constraints has been debated as a benefit and hinderance to levels of innovation in the development process. In a study by Hatchel and Chen [8] explored the effects of constraints on the development of new products using concept knowledge theory (C-K Theory). Within their study it was found that the levels of innovation in a new product development were directly linked to the designer's ability to challenge the identity and heritage of the product. In the case of the project brief students were expected to follow the fundamental functionality of the Potters for peace (PFP) style filtration system [9], while leaving the cultural heritage of the object open to interpretation. This was intended to allow for a greater range of exploration and experimentation between the students. The cultures selected by each student can be viewed in Table 1.

Table 1. Students and their target cultures for product development

\begin{tabular}{|l|l|l|l|l|l|l|}
\hline Student: & A & B & C & D & E & F \\
\hline $\begin{array}{l}\text { Region of } \\
\text { choice: }\end{array}$ & $\begin{array}{l}\text { Brazil } \\
\text { (Favelas) }\end{array}$ & Tibet & Ethiopia & Ghana & Sri Lanka & $\begin{array}{l}\text { India } \\
\text { (Mumbai) }\end{array}$ \\
\hline
\end{tabular}

\subsection{Concept generation and production}

Students used a mixture of traditional and modern production methods ranging from coiling, press moulding, slab building and turning to produce their initial concepts. Where students had difficulty using the coiling technique to produce uniform profiles, they were encouraged to use plaster moulds as guides to produce the base of their concepts. This is a common practice in African pottery crafts, where instead of plaster moulds, curved pieces of broken ceramics or rocks may be used as a mould or anvil for the shaping of clay walls [10].

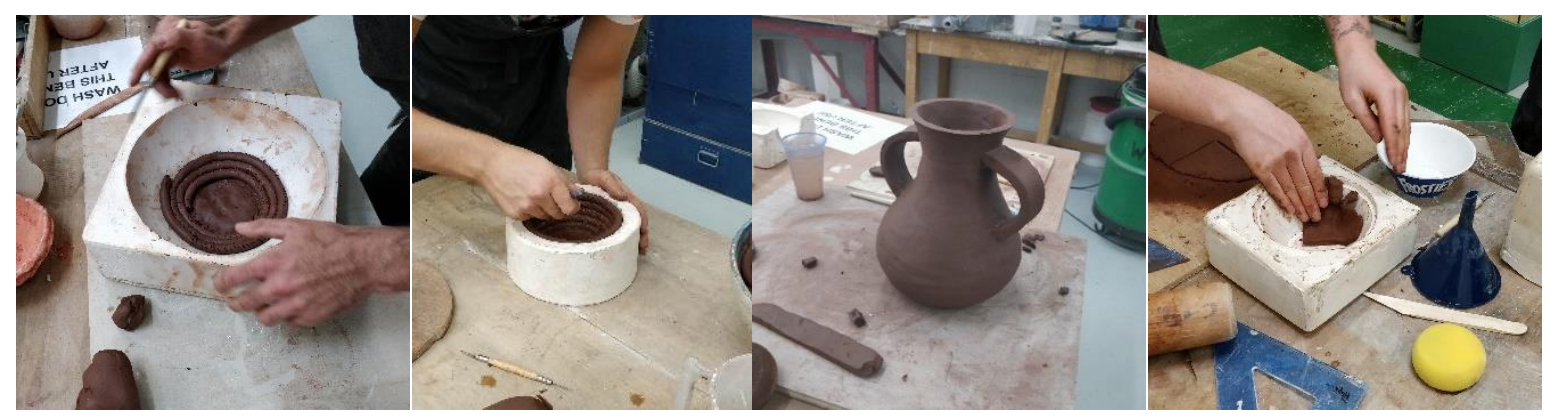

Coiling

Press Moulding

Figures 1-4. Far left: Image of pot base constructed using coiling. Left: Image of pot base being constructed using coiling. Right: Tibetan inspired vessel, constructed using press moulding. Far right: Filter clay mixture being press moulded into plaster mould

To achieve the final styling of their design, students took moulds from a range of existing, and custom forms. Each of these forms were cast in plaster with units coiled or press moulded into them to produce their final shapes. Where existing forms were not available, or where students had very specific requirements for their concept designs, CAD models were generated and either 3D printed for use in mould casting, or as guides for turning forms on the lathe or potters' wheel. An example produced by Student B can be seen in the figures 5-7. 


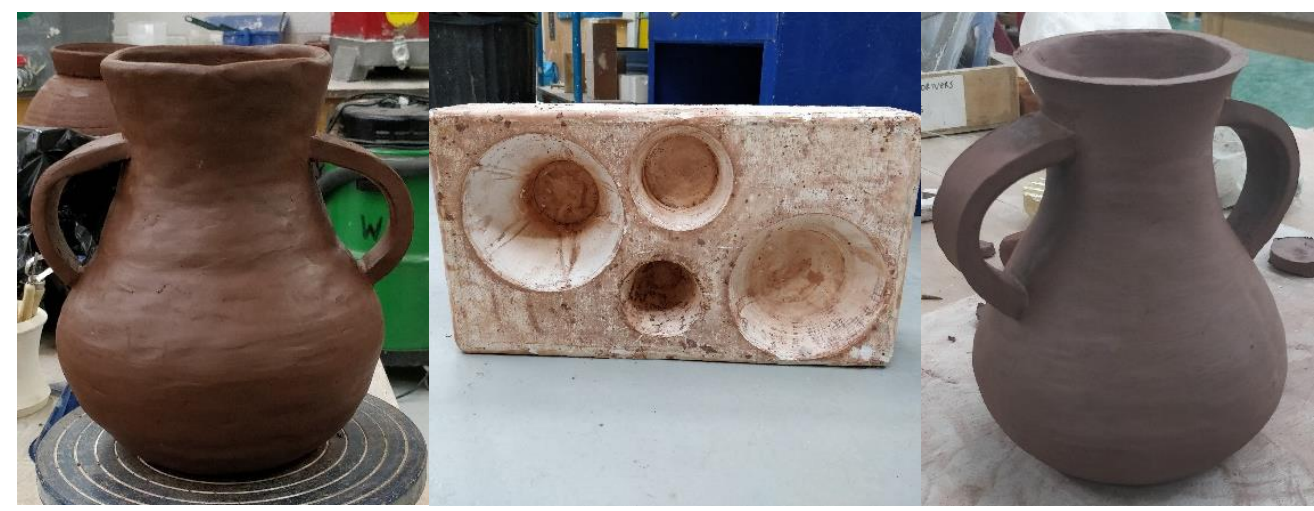

Figures 5-7. Left: Student B pot constructed using coiling technique. Centre: Mould produced using 3D printed forms. Right: Vessel produced from press moulded clay

Student B originally produced their design using the coiling method (showing in figure 5) but felt that the outcome did not suitably match the finish specified in the design. The design was broken down into a series of forms able to be produced using a one-part dropout mould, and then 3D printed. Each section was produced using a two-layer thick $(0.8 \mathrm{~mm}$ wall thickness $)$ printed shell that could be easily moulded and removed. A single mould was produced incorporating three pieces making up the main body of the vessel and one additional form to produce the filter (see figure 6). The sections were press moulded and then constructed to produce the final form (see figure 7). The same process was used for the turned forms produced by student $\mathrm{A}$ and $\mathrm{E}$.

\subsection{Filter production}

Filters were produced using the method suggested by PFP [9], using a combination of sawdust (harvested from the furniture workshop) and powdered terracotta clay (other clays were provided, though terracotta best suited the cultural demographics of the students' selected countries). The clay was mixed with the sawdust in a powdered form to prevent water content from creating an uneven ratio of clay to sawdust. Students used a 1:1 ratio of powdered clay to sawdust and were given examples of fired filter units made with 1:1, 1:1.5 and 1:2 ratios of clay to saw dust. This enabled the students to view the different flow rates, strengths and textures that resulted from the different ratios. Students also commented on the differences between the raw filter clay and the standard terracotta, expressing that the filter clay was softer and easier to break while shaping it. The texture and malleability of these clay mixtures are not easily conveyed without manual manipulation, and as such were a valuable experience in the student's development process.

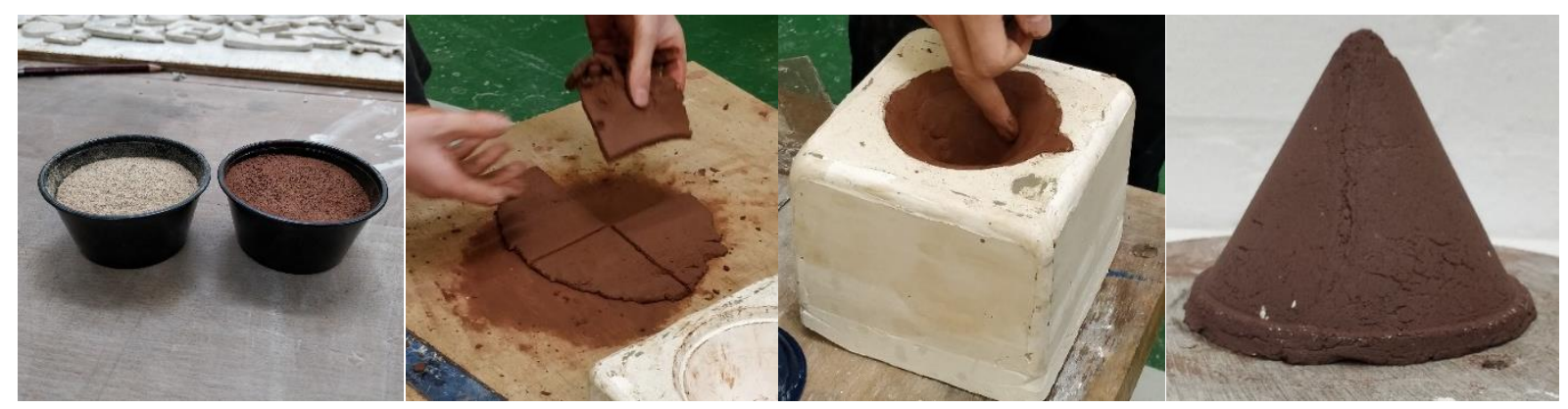

Figures 8-11. Far left: Equal volumes of sawdust and powdered terracotta. Left: filter clay mixture prepared for press moulding. Right: Filter clay mixture moulded in plaster a plaster mould. Far right: Dried filter clay part pre-firing

\subsection{Glazing and finishing}

Some of the finishes required by the student's concepts were not possible to create in the university's electric kilns. Many of the designs required either gas fed, or outdoor pit or bonfire kilns to achieve the traditional finishes familiar to their intended users. To remedy this constraint students were instructed in the production of a base alkaline glaze composed of high alkaline frit, flint and china clay that colorants could be added to. While this was not an ideal solution to produce fully representative products 
as they would be produced in the intended area of adoption, it provided the students with an understanding of the regional and industrial composition of ceramic finishes. Depending on the design features used, students used a range of glazing techniques including dip glazing, brush glazing and spray glazing (See figures 12-14).

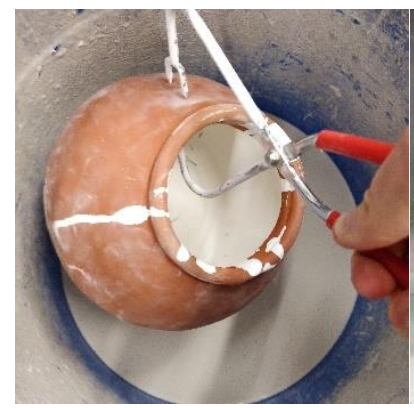

Dip glazing

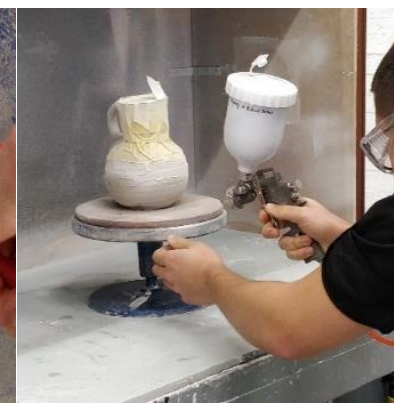

Spray glazing

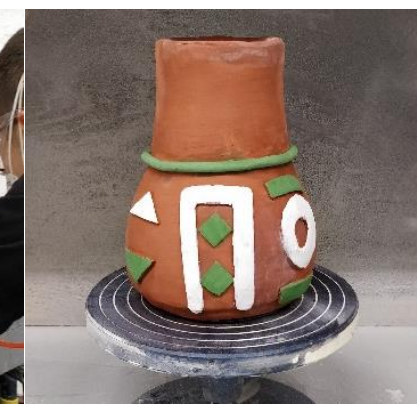

Brush glazing

Figures 12-14. Left: Pot produced by student $F$ being dip glazed in a clear bisilicate glaze. Centre: Student E spray glazing Sri Lankan inspired vessel. Right: Student $A$ vessel with brush glazed coloured embellishments

\section{CONCLUSIONS}

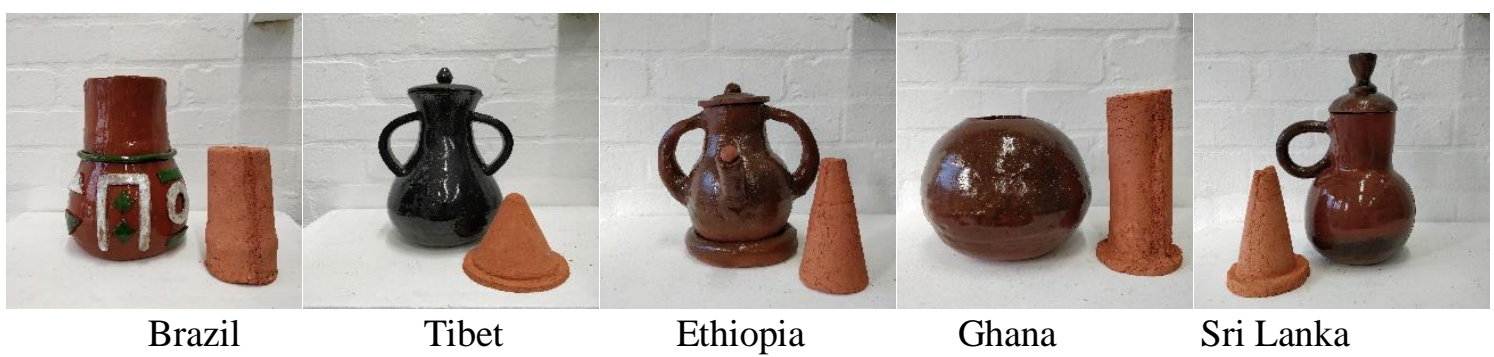

Figures $15-19$. Images of the final filters and vessels produced by the level five group

The primary aim of this module was to introduce students to user centred design considerations in the development of a water filtration system for a developing area. Students engaged well with the subject matter, taking suitable inspiration from their selected regions and implementing them into their designs. In applying the three levels of processing to the concepts produced, each did show clear understanding of aesthetic, functional and cultural perspectives. From a technical perspective each unit was capable of adequately filtering water and collecting it within their storage vessels. Observing the finished models from a usability perspective, the weight of each container was well managed allowing for easy handling by users with a range of abilities and different stages of development. Despite the reasonably short project timeframe, the students were able to apply a range of ceramic manufacturing methods effectively. Their application of craft and contemporary technologies produced fully working concepts, accounting correctly for the shrinkage rates of the differing clays used. Having completed a ceramic project as a part of their level four manufacturing module the previous year, the students commented positively on the project brief, preferring the technical and user centred focus over the more artistic exploration of form.

On reflection the management of water post process required further communication during the module. All but one of the student's final concepts did not incorporate a method of retrieving the filtrate from the container, other than pouring the contents from the containers point of entry. In a real-world scenario, this would significantly increase the chances of the recontamination of the filtrate and risk contamination or breakage of the filter from handling outside of the vessel. While these issues were raised during the lectures and workshop sessions, the students would have benefited from a greater level of technical information and context to contribute to their understanding of the end users' specific requirements. The level of constraints applied to the student's assignment were originally intended to allow for innovation in the design of the filter and containment vessels form and usability. Having reviewed the final design outcomes there appears to have been a prevalent incidence of students focusing on the replication of 
traditional ceramics rather than adaptation of traditional styles. Here the points raised by Hatchel and Chen [8] may have been inadvertently addressed twice, constricting the students design outcome not only by the technological heritage of the PFP technology, but also by the cultural design styles of the end user. It is recommended that future projects place greater emphasis on the concept generation stage of the project with a greater leniency on challenging the conventions of the technology and design style. This change will ensure that a wider range of solutions are explored and allowing for a greater opportunity for innovation.

\section{REFERENCES}

[1] Rognoli V. A broad survey on expressive-sensorial characterization of materials for design education. Journal of the Faculty of Architecture, 2010, 27:2, 287-300.

[2] Shin D. and Chung S. Tactile Digital: An exploration of merging ceramic art and industrial design. International conference on engineering and product design education, Aalborg, September 2016, pp.688-693.

[3] Norman D. The design of everyday things, Revised edition, 2013 (Basic Books) pp.50-53

[4] Harlow R. and Buck L. Improvements in water intervention projects through product design methods. International conference on engineering and product design education, Glasgow, September 2019,

[5] Pedgley O. Rognoli V. and Karana E. Materials experience as a foundation for materials and design education. International journal for technology design education, 2016, 26:616-630

[6] Papanek V. Design for the Real World, 1971 (Academy Chicago Publishers, Chicago) pp.69

[7] Kang L. Social design as a creative device in developing countries: The case of a handcraft pottery community in Cambodia. International journal of design, 2016, 10:3, 65-74

[8] Hatchuel A. and Chen M K. Creativity under strong constraints: the hidden influence of design models traduction. 2017 (European Review, Cambridge University Press).

[9] Potters for peace. Best practice recommendations for local manufacturing of ceramic pot filters for household water treatment. Available: https://b06e35ce-2a1a-4f6b-935c3a97c8f0fbb7.filesusr.com/ugd/2802c0_91b40a48e8a348e5989aa91b6d2d8998.pdf [Accessed on 2020, 10,01] (2011) June.

[10] Barley N. Smashing pots, Feats of clay from Africa. 1994 (Smithsonian institution press) pp.2429.

The WATERSPOUTT project described in this paper has received funding from the European Union's Horizon 2020 research and innovation programme under grant agreement number 688928 